\begin{tabular}{|c|l|}
\hline Title & Lyapunov functions and global stability for SIR and SIRS epidemiological models with non-linear transmission \\
\hline Author(s) & Korobeinikov, A ndrei \\
\hline Citation & $\begin{array}{l}\text { Bulletin of Mathematical Biology, 68/3), 615-626 } \\
\text { https://doi.org/10.1007/311535-005-9037-9 }\end{array}$ \\
\hline Issue Date & 2006-04 \\
\hline Doc URL & http://hdl.handle.net/2115/33886 \\
\hline Rights & The original publication is available at www.springerlink.com \\
\hline Type & article (author version) \\
\hline File Information & paper2.pdf \\
\hline
\end{tabular}

Instructions for use 


\title{
LYAPUNOV FUNCTIONS AND GLOBAL STABILITY FOR SIR AND SIRS EPIDEMIOLOGICAL MODELS WITH NON-LINEAR TRANSMISSION
}

\author{
ANDREI KOROBEINIKOV
}

Laboratory of Nonlinear Science and Computation

Research Institute for Electronic Science, Hokkaido University,

Sapporo 060-0812, Japan

andrei@nsc.es.hokudai.ac.jp

phone (81 11) 7062414

fax (81 11) 7064966

\section{AMS Classification \\ 92D30 (primary), 34D20 (secondary)}

\section{Proposed Running Head}

Lyapunov functions for SIR and SIRS models 
Abstract. Lyapunov functions for 2-dim SIR and SIRS compartmental epidemic models with non-linear transmission rate of a very general form $f(S, I)$ constrained by a few biologically feasible conditions are constructed. Global properties of these models including these with vertical and horizontal transmission, are thereby established. It is proved that, under the constant population size assumption, the concavity of the function $f(S, I)$ with respect to the number of the infective hosts $I$ ensures the uniqueness and the global stability of the positive endemic equilibrium state.

Key words: Direct Lyapunov method, Lyapunov function, endemic equilibrium state, global stability, nonlinear incidence.

Acknowledgement. Work is supported by Japan Society for the Promotion of Science, through Project 17540099.

\section{INTRODUCTION}

Classical epidemiological models postulate that the spread of an infection occurs according to the principle of mass action and associated with that an incidence rate bilinear with respect to the numbers of susceptible and infective individuals (Anderson and May, 1991; Hethcote, 2000). More specifically, if $S(t)$ and $I(t)$ are the fractions of susceptibles and infective individuals in a population, and if $\beta$ is the per capita contact rate, then the infection spreads with the rate $\beta I S$. However, there is a variety of reasons why this standard bilinear incidence rate may require modification. For example, the underlying assumption of homogeneous mixing may be invalid. In this case the necessary population structure and heterogeneous mixing may be incorporated into a model with a specific form of non-linear transmission. A nonlinear incidence rate also arises from saturation effects: if the proportion of 
the infective hosts in a population is very high, so that exposure to the disease agent is virtually certain, then the transmission rate may respond more slowly than linear to the increase in the number of infectives. This effect was observed, for example, by Capasso and Serio (1978) who studied the cholera epidemic spread in Bari in 1973, and by Brown and Hasibuan (1995) who studied infection of the two-spotted spider mites, Tetranychus urticae, with the entomopathogenic fungus, Neozygites floridana.

In recent years, a number of authors have studied epidemiological models with nonlinear incidence rates. The most common nonlinear incidence rate takes the form $\beta I^{p} S^{q}$ (where $p$ and $q$ are positive constants). Models with this incidence rate have been studied by Liu et al. (1986, 1987), and later by Hethcote et al. (1989); Hethcote and van den Driessche (1991); Derrick and van den Driessche (1993, 2003); Korobeinikov and Maini (2004) and many others. The nonlinear incidence rates of more sophisticated forms of were also suggested. For instance, Liu et al. (1986) proposed an incidence rate of the form $k I^{p} S /\left(1+\alpha I^{l}\right)$; Briggs and Godfray (1995), who studied infection of insects, considered a non-linear pathogen transmission of the form $k S \ln (1+\nu P / k)$ (where $P$ is the density of the pathogen particles). Li et al. (1999) studied the global properties of a SEIR model with the incidence rate of the form $g(I) S$; they also extended some of their results for a $S E I R S$ model. Korobeinikov and Maini (2005) considered a variety of models with the incidence rate of the form $g(I) h(S)$; constructed Lyapunov functions enabled to establish global properties for a $S I R$ and a $S E I R$ models. The most general case was considered by Feng and Thieme (2000,a), who considered properties of a model with arbitrarily many 
stages of infection, all of which have general length distributions, and with incidence rate of a very general form. They found the condition for existence of a unique endemic equilibrium state and for its local stability.

In this note we attempt to extend the Lyapunov functions constructed earlier (Korobeinikov and Maini, 2005) to a more general incidence rate given by an arbitrary function $f(S, I)$. We prove that the concavity of the incidence rate with respect to the number of the infective hosts leads to a globally stable system. We have to note that the global stability of the models considered in this paper was long con-

jectured and for some particular forms of incidence rate has already been established. The direct Lyapunov method enables us to obtain this result straightforwardly and for a very general form of incidence rate.

\section{SIR MODEL}

Following classic assumptions (Anderson and May, 1991; Busenberg and Cooke, 1993; Hethcote, 2000), we divide a host population of a constant size into susceptible, infective and recovered subpopulations and denote the fractions of these in the population by $S, I$ and $R$ respectively (that is $S+I+R=1$ ). After infection an individual moves from the susceptibles compartment into the infectives compartment and then into the recovered compartment. We assume that recovery implies permanent immunity. A model based on these assumptions is known as a $S I R$ model. 
We assume that the population size is constant, that is deaths are balanced by births, and that the transmission of the infection is governed by an incidence rate $f(S, I)$. Then the basic $S I R$ model is

$$
\begin{aligned}
\dot{S} & =\mu-f(S, I)-\mu S, \\
\dot{I} & =f(S, I)-\delta I .
\end{aligned}
$$

Here $\mu$ is the death/birth rate and $\delta$ is the sum of the death rate of infected individuals (which is here assumed to be equal to the death rate of susceptibles) and the recovery rate. The equation for the recovered population, which in this case is

$$
\dot{R}=(\delta-\mu) I-\mu R
$$

is usually omitted - the constant population size assumption enables us to do that.

Naturally, $f(S, I)$ is a positive and monotonically growing function for all $S, I>0$, and $f(0, I)=f(S, 0)=0$. These conditions arise solely from biological considerations; further we will show that they are not necessary for our analysis.

It is easy to see that the non-negative quadrant of the $S I$ plane is an invariant set of the system. The system has two equilibrium states: an infection-free equilibrium $Q_{0}$ with the coordinates $S_{0}=1, I_{0}=0$, and an endemic equilibrium state $Q^{*}=\left(S^{*}, I^{*}\right)$, such that

$$
\mu=f\left(S^{*}, I^{*}\right)+\mu S^{*} \text { and } \delta I^{*}=f\left(S^{*}, I^{*}\right)
$$

We will show that, under certain biologically reasonable conditions, if the function $f(S, I)$ is concave with respect to the variable $I$ (that is 
if $\frac{\partial^{2} f}{\partial I^{2}} \leq 0$ holds for all $I>0$ ), then the uniqueness of the equilibrium state $Q^{*}$ is ensured.

For this model, the basic reproduction number, that is an average number of secondary cases produced by a single infective introduced into entirely susceptible population, is (van den Driessche and Watmough, 2002)

$$
R_{0}=\frac{1}{\delta} \frac{\partial f\left(S_{0}, I_{0}\right)}{\partial I}
$$

Theorem 2.1. (i) If the function $f(S, I)$ monotonically grows with respect to $S$ and $I$ and is concave with respect to the variable $I$ (that is

if $\frac{\partial^{2} f}{\partial I^{2}} \leq 0$ ), and if $R_{0}>1$, then the system (2.1) has an unique positive endemic equilibrium state $Q^{*}$ which is globally asymptotically stable.

(ii) If $R_{0} \leq 1$, then there is no positive endemic equilibrium state, and the infection-free equilibrium state $Q_{0}$ is globally asymptotically stable.

Proof. 1. Existence of an positive equilibrium state. At a fixed point of the system the equalities $\delta I+\mu S=\mu$ and $\delta I=f(S, I)$ hold. These equalities define a negatively sloped straight line $q_{1}$ and a curve $q_{2}$ on the $I S$ plane (Fig. 2.1). The equality $\delta I=f(S, I)$ defines also a function $S=h(I)$. If $\frac{\partial f(S, I)}{\partial S}$ is strictly positive, then, by the implicit function theorem, the function $h(I)$ is defined and continuous for all $I>0$. It is obvious (see Fig. 2.1) that if $S_{*}=h(0) \leq S_{0}=1$ then there is at least one pont of intersection of the lines $q_{1}$ and $q_{2}$. The function $f(S, I)$ grows monotonically with respect to both its variables, and hence $S_{0} / S_{*}>1$ if

$$
\lim _{I \rightarrow 0} \frac{f\left(S_{0}, I\right)}{f\left(S_{*}, I\right)}=\lim _{I \rightarrow 0} \frac{f\left(S_{0}, I\right)}{\delta I}=\frac{1}{\delta} \frac{\partial f\left(S_{0}, 0\right)}{\partial I}=R_{0}>1 .
$$




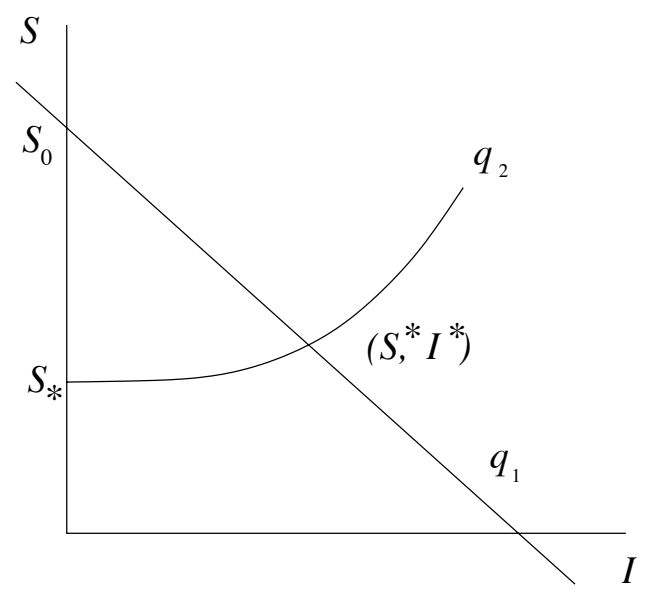

FiguRE 2.1. The straight line $q_{1}$ and the curve $q_{2}$.

2. Stability of the endemic equilibrium state. We assume that $R_{0}>1$, and hence there exists a positive endemic equilibrium state $Q^{*}=\left(S^{*}, I^{*}\right)$. A function

$$
V(S, I)=S-\int_{\varepsilon}^{S} \frac{f\left(S^{*}, I^{*}\right)}{f\left(\tau, I^{*}\right)} d \tau+I-\int_{\varepsilon}^{I} \frac{f\left(S^{*}, I^{*}\right)}{f\left(S^{*}, \tau\right)} d \tau .
$$

is defined and continuous for all $S, I>\varepsilon$. If $f(S, I)$ is monotonic with respect to both variables, then the endemic equilibrium state $Q^{*}$ is the only extremum and the global minimum of this function. Indeed,

$$
\frac{\partial V}{\partial S}=1-\frac{f\left(S^{*}, I^{*}\right)}{f\left(S, I^{*}\right)}, \quad \frac{\partial V}{\partial I}=1-\frac{f\left(S^{*}, I^{*}\right)}{f\left(S^{*}, I\right)}
$$

and hence $Q^{*}=\left(S^{*}, I^{*}\right)$ is a stationary point of the function. Since the function $f(S, I)$ monotonically grows with respect to both its variables, the partial derivatives $\frac{\partial V}{\partial S}$ and $\frac{\partial V}{\partial I}$ monotonically grow as well, and hence $Q^{*}$ is the only stationary point. Furthermore, since

$$
\frac{\partial^{2} V}{\partial S^{2}}=\frac{f\left(S^{*}, I^{*}\right)}{\left(f\left(S, I^{*}\right)\right)^{2}} \frac{\partial f\left(S, I^{*}\right)}{\partial S} \quad \text { and } \quad \frac{\partial^{2} V}{\partial I^{2}}=\frac{f\left(S^{*}, I^{*}\right)}{\left(f\left(S^{*}, I\right)\right)^{2}} \frac{\partial f\left(S^{*}, I\right)}{\partial I}
$$

are both non-negative, the point $Q^{*}$ is a minimum. That is $V(S, I) \geq$ $V\left(S^{*}, I^{*}\right)$. Consequently, the function $V(S, I)$ is a Lyapunov function. 
In the case of the system (2.1), using

$$
\mu=f\left(S^{*}, I^{*}\right)+\mu S^{*} \quad \text { and } \quad \delta I^{*}=f\left(S^{*}, I^{*}\right)
$$

(cf. (2.2)), the Lyapunov function (2.3) satisfies

$$
\begin{aligned}
\frac{d V(S, I)}{d t}= & \mu-f(S, I)-\mu S-\mu \frac{f\left(S^{*}, I^{*}\right)}{f\left(S, I^{*}\right)}+\frac{f\left(S^{*}, I^{*}\right)}{f\left(S, I^{*}\right)} f(S, I)+\mu S \frac{f\left(S^{*}, I^{*}\right)}{f\left(S, I^{*}\right)} \\
& +f(S, I)-\delta I-\frac{f\left(S^{*}, I^{*}\right)}{f\left(S^{*}, I\right)} f(S, I)+\delta I \frac{f\left(S^{*}, I^{*}\right)}{f\left(S^{*}, I\right)} \\
= & \mu S^{*}\left(1-\frac{S}{S^{*}}-\frac{f\left(S^{*}, I^{*}\right)}{f\left(S, I^{*}\right)}+\frac{S}{S^{*}} \frac{f\left(S^{*}, I^{*}\right)}{f\left(S, I^{*}\right)}\right) \\
& +f\left(S^{*}, I^{*}\right)\left(1-\frac{f\left(S^{*}, I^{*}\right)}{f\left(S, I^{*}\right)}-\frac{f(S, I)}{f\left(S^{*}, I\right)}\right) \\
& +f\left(S^{*}, I^{*}\right)\left(-\frac{I}{I^{*}}+\frac{f(S, I)}{f\left(S, I^{*}\right)}+\frac{I}{I^{*}} \frac{f\left(S^{*}, I^{*}\right)}{f\left(S^{*}, I\right)}\right) \\
= & \mu S^{*}\left(1-\frac{S}{S^{*}}\right)\left(1-\frac{f\left(S^{*}, I^{*}\right)}{f\left(S, I^{*}\right)}\right) \\
& +f\left(S^{*}, I^{*}\right)\left(1-\frac{f\left(S^{*}, I^{*}\right)}{f\left(S, I^{*}\right)}\right)\left(1-\frac{f(S, I)}{f\left(S^{*}, I\right)}\right) \\
& +f\left(S^{*}, I^{*}\right)\left(\frac{I}{I^{*}}-\frac{f(S, I)}{f\left(S, I^{*}\right)}\right)\left(\frac{f\left(S^{*}, I^{*}\right)}{f\left(S^{*}, I\right)}-1\right) .
\end{aligned}
$$

It is easy to see that if $Q^{*}>0$ and the function $f(S, I)$ is concave with respect to $I$ (that is, if $\frac{\partial^{2} f(S, I)}{\partial I^{2}} \leq 0$ holds), then $\frac{d V}{d t} \leq 0$ for all $S, I>0$. Indeed, the monotonicity of $f(S, I)$ with respect to $S$ ensures that

$$
\left(1-\frac{S}{S^{*}}\right)\left(1-\frac{f\left(S^{*}, I^{*}\right)}{f\left(S, I^{*}\right)}\right) \leq 0
$$

and

$$
\left(1-\frac{f\left(S^{*}, I^{*}\right)}{f\left(S, I^{*}\right)}\right)\left(1-\frac{f(S, I)}{f\left(S^{*}, I\right)}\right) \leq 0
$$

hold for all $S, I>0$. Furthermore,

$$
\left(\frac{I}{I^{*}}-\frac{f(S, I)}{f\left(S, I^{*}\right)}\right)\left(\frac{f\left(S^{*}, I^{*}\right)}{f\left(S^{*}, I\right)}-1\right) \leq 0
$$




$$
\begin{aligned}
f(S, I) / f\left(S, I^{*}\right) & \geq I / I^{*}, \text { when } \quad f\left(S^{*}, I\right) \leq f\left(S^{*}, I^{*}\right), \text { and } \\
(2.5) f(S, I) / f\left(S, I^{*}\right) & \leq I / I^{*}, \quad \text { when } \quad f\left(S^{*}, I\right) \geq f\left(S^{*}, I^{*}\right)
\end{aligned}
$$

holds for all $S, I>0$. For a monotonic function, $f\left(S^{*}, I\right) \geq f\left(S^{*}, I^{*}\right)$

implies $I \geq I^{*}$, and $f\left(S^{*}, I\right) \leq f\left(S^{*}, I^{*}\right)$ implies $I \leq I^{*}$, and hence the condition (2.5) is equivalent to the condition

$$
\begin{aligned}
& f(S, I) / f\left(S, I^{*}\right) \geq I / I^{*} \text { for all } I \leq I^{*}, \text { and } \\
& f(S, I) / f\left(S, I^{*}\right) \leq I / I^{*} \text { for all } I \geq I^{*} .
\end{aligned}
$$

It is obvious, that this condition (and hence the condition (2.5)) holds for all functions concave with respect to the variable $I$ (see Fig. 2.2), and hence the concavity is sufficient to ensure that $\frac{d V(S, I)}{d t} \leq 0$ in $\mathbf{R}_{+}^{2}$.

\section{Uniqueness of the positive endemic equilibrium state. We} assume now that apart from the equilibrium $Q^{*}$, the system has another positive equilibrium state $Q_{1}=\left(S_{1}, I_{1}\right)$. Then $f\left(S_{1}, I_{1}\right)+\mu S_{1}=\mu$ and $\delta I_{1}=f\left(S_{1}, I_{1}\right)$ hold. The derivative of a Lyapunov function is equal to zero at any equilibrium state, and therefore $\frac{d V}{d t}=0$ at $Q_{1}$. Therefore, $S_{1}$ and $I_{1}$ must satisfy the equalities

$$
\begin{aligned}
\left(1-\frac{S_{1}}{S^{*}}\right)\left(1-\frac{f\left(S^{*}, I^{*}\right)}{f\left(S_{1}, I^{*}\right)}\right) & =0, \\
\left(1-\frac{f\left(S^{*}, I^{*}\right)}{f\left(S_{1}, I^{*}\right)}\right)\left(1-\frac{f\left(S_{1}, I\right)}{f\left(S^{*}, I\right)}\right) & =0, \\
\left(\frac{I_{1}}{I^{*}}-\frac{f\left(S_{1}, I_{1}\right)}{f\left(S_{1}, I^{*}\right)}\right)\left(\frac{f\left(S^{*}, I^{*}\right)}{f\left(S^{*}, I_{1}\right)}-1\right) & =0 .
\end{aligned}
$$




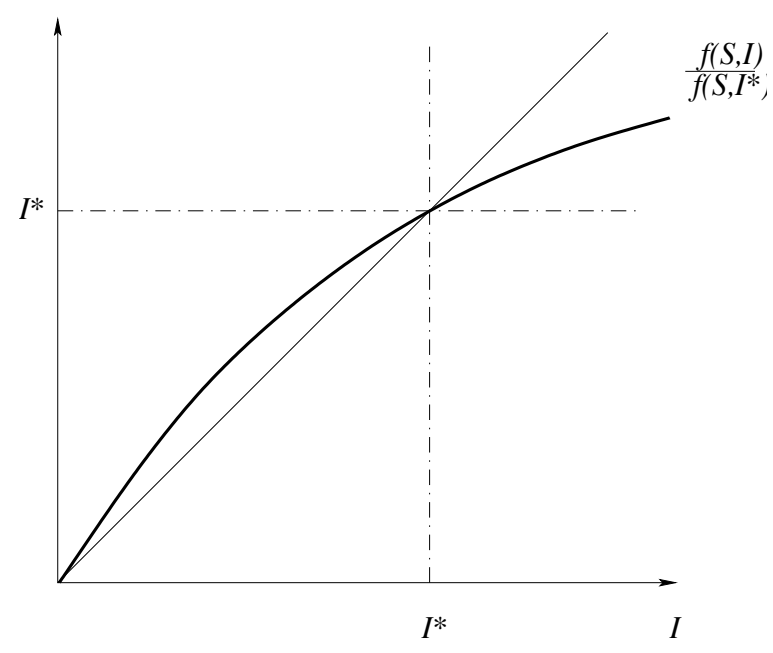

Figure 2.2. A function $f(S, I)$ concave with respect to $I$.

It is obvious that for a monotonic function (or for a function satisfying condition (2.4)), the equality (2.6) holds only when $S_{1}=S^{*}$. Then $I_{1}=I^{*}$ is necessary to satisfy $f\left(S^{*}, I_{1}\right)+\mu S^{*}=\mu$. That is $S_{1}=S^{*}$ and $I_{1}=I^{*}$, and hence $Q^{*}$ is the only positive fixed point of the system.

Furthermore, it is also obvious that for a monotonic (or satisfying condition (2.4)) function, the point $Q^{*}$ is the only invariant set of the system (2.1) in the set $\frac{d V}{d t}=0$. Therefore, by the Lyapunov-La Salle principle (Barbashin, 1970; La Salle and Lefschetz, 1961), the point $Q^{*}$ is asymptotically stable for all $S \geq \varepsilon$.

The parameter $\varepsilon$ may be made as small as required, and therefore the endemic equilibrium $Q^{*}$ is asymptotically stable in the non-negative quadrant $\mathbf{R}_{+}^{2}$.

4. Stability of the infection-free equilibrium state. To prove the global stability of the infection-free equilibrium states $Q_{0}=(1,0)$ we consider the Lyapunov function

$$
U(S, I)=S-\int_{\varepsilon}^{S} \lim _{\substack{I \rightarrow I_{0} \\ 10}} \frac{f\left(S_{0}, I\right)}{f(\tau, I)} d \tau+I .
$$


(We cannot consider the function $U=S-\int_{\varepsilon}^{S} \frac{f\left(S_{0}, I_{0}\right)}{f\left(\tau, I_{0}\right)} d \tau+I$, because $f(S, 0)=0$.) In the case of the $S I R$ system (2.1), the Lyapunov function satisfies

$$
\begin{aligned}
\frac{d U(S, I)}{d t}= & \mu-f(S, I)-\mu S-\mu \lim \frac{f\left(S_{0}, I_{0}\right)}{f\left(S, I_{0}\right)}+f(S, I) \lim \frac{f\left(S_{0}, I_{0}\right)}{f\left(S, I_{0}\right)} \\
& +\mu S \lim \frac{f\left(S_{0}, I_{0}\right)}{f\left(S, I_{0}\right)}+f(S, I)-\delta I \\
= & \mu-\mu \frac{S}{S_{0}}-\mu \lim \frac{f\left(S_{0}, I_{0}\right)}{f\left(S, I_{0}\right)}+f(S, I) \lim \frac{f\left(S_{0}, I_{0}\right)}{f\left(S, I_{0}\right)}+\mu \frac{S}{S_{0}} \lim \frac{f\left(S_{0}, I_{0}\right)}{f\left(S, I_{0}\right)}-\delta I \\
= & \mu\left(1-\frac{S}{S_{0}}\right)\left(1-\lim \frac{f\left(S_{0}, I_{0}\right)}{f\left(S, I_{0}\right)}\right)+\delta I\left(\frac{f(S, I)}{\delta I} \lim \frac{f\left(S_{0}, I_{0}\right)}{f\left(S, I_{0}\right)}-1\right) .
\end{aligned}
$$

(Here we denote $\lim \frac{f\left(S_{0}, I_{0}\right)}{f\left(S, I_{0}\right)}=\lim _{I \rightarrow I_{0}} \frac{f\left(S_{0}, I\right)}{f(S, I)}$.) For a monotonic function

$$
\left(1-\frac{S}{S_{0}}\right)\left(1-\lim \frac{f\left(S_{0}, I_{0}\right)}{f\left(S, I_{0}\right)}\right) \leq 0 \text { for all } S>0
$$

Concavity of the function $f(S, I)$ ensures that $f(S, I) \leq I \frac{\partial f(S, 0)}{\partial I}$ for all $I>0$, and hence

$$
\frac{f(S, I)}{\delta I} \lim _{I \rightarrow I_{0}} \frac{f\left(S_{0}, I\right)}{f(S, I)}=\frac{f(S, I)}{\delta I} \frac{\frac{\partial f\left(S_{0}, I_{0}\right)}{\partial I}}{\frac{\partial f\left(S, I_{0}\right)}{\partial I}} \leq \frac{1}{\delta} \frac{\partial f\left(S_{0}, I_{0}\right)}{\partial I}=R_{0} .
$$

Therefore, $R_{0} \leq 1$ ensures that $\frac{d U(S, I)}{d t} \leq 0$ for all $S, I>\varepsilon$, and hence by the asymptotic stability theorem (Barbashin, 1970; La Salle and Lefschetz, 1961) the equilibrium state $Q_{0}$ is globally asymptotically stable in this case.

We proved that for all $R_{0}>1$ there exists an unique and globally stable positive equilibrium state $Q^{*}$, and that for $R_{0}<1$ the infectionfree equilibrium $Q_{0}$ is globally stable. At $R_{0}=1$ for any function $f(S, I)$ monotonic with respect to $S$ these two equilibria, $Q_{0}$ and $Q^{*}$, meet and exchange their stability; that is a transcritical bifurcation 
occurs at this point. Indeed, it is easy to see (cf. Proof, Part 1) that, for a monotonic function $f(S, I), S_{*}$ (and hence $S^{*}$ ) tends to $S_{0}$ as $R_{0}$ tends to 1 , and that $R_{0}=1$ implies $S_{*}=S_{0}$. At $R_{0}=1$ the point $\left(S_{0}, I_{0}\right)$ is, therefore, the point of intersection of the lines $q_{1}$ and $q_{2}$ (see Fig. 2.1). For $R_{0}<1$ the equilibrium $Q^{*}$ moves in the quadrant $S>0, I<0$.

\section{SIRS MODEL}

The $S I R$ model postulates permanent immunity of the recovered individuals. However, for some infections this acquired immunity can disappear after some time. A SIRS model describes such a case.

A further complication of the model is to assume a possibility for a so-called "vertical transmission", that is transplacental transmission from an infective mother to an unborn or newly born child (Busenberg and Cooke, 1993). To incorporate the vertical transmission into a $S I R$ or a SIRS model we assume that a fraction $p$ of offsprings of the infective hosts is infected at birth, and hence a part of birth flux, $p \mu I$, enters the infective compartment while the remaining births, $\mu(1-p) I$, come to the susceptibles compartment (Busenberg and Cooke, 1993). Then the SIRS model equations are

$$
\begin{aligned}
\dot{S} & =(\mu+\alpha)-f(S, I)-\alpha I-p \mu I-(\mu+\alpha) S \\
\dot{I} & =f(S, I)+p \mu I-\delta I .
\end{aligned}
$$

Here $1 / \alpha$ is an average period of immunity. We assume as before that $\delta$ is the sum of the death rate and the recovery rate of the infective hosts, and $0 \leq p \leq 1$, hence $\delta>p \mu$. The equation for the recovered population $R$ is omitted. 
It is easy to see that the positive quadrant of $S I$ plane is not an invariant set of this system. Indeed, $\dot{S}<0$ at $S=0$ for all $I>$ $(\mu+\alpha) /(p \mu+\alpha)$. Therefore, the problem of proving the global stability for the whole positive quadrant is meaningless in this case. However, such a proof is possible for the so-called "feasible region"

$$
\Sigma=\left\{(S, I) \in \mathbf{R}^{2} \mid S, I>0 ; S+I \leq 1\right\},
$$

which is usually considered as the phase space of the system. This region is an invariant set of the system (it is easy to see that the phase flow through all its boundaries is directed inward), and that the positive endemic equilibrium state belong to this region, when it exists.

Assume that the function $f(S, I)$ is continuous and defined for $S<0$. Denoting $\widetilde{\mu}=\mu+\alpha, \widetilde{\delta}=\delta+\alpha$ and $\widetilde{f}(S, I)=f(S, I)+(\alpha+p \mu) I$, we obtain the equations

$$
\begin{aligned}
\dot{S} & =\widetilde{\mu}-\widetilde{f}(S, I)-\widetilde{\mu} S, \\
\dot{I} & =\widetilde{f}(S, I)-\widetilde{\delta} I .
\end{aligned}
$$

We assume that the phase space of this system is the region $\Omega \in \mathbf{R}^{2}$ such that $\tilde{f}(S, I)>0$ for all $(S, I) \in \Omega$. For a biologically feasible transmission rate, $f(S, I)>0$ for all $S, I>0$, and $f(0, I)=0$, and therefore $\Sigma \subset \mathbf{R}_{+}^{2} \subset \Omega$.

It is obvious that the systems (2.1) and (3.2) are equivalent. Therefore, Theorem 2.1 holds for system (3.2) as well: the only alteration needed is that for the system (3.2) the global stability holds in the region $\Omega$. (We need to take care about the lower limit for the first integral of function (2.3); however, that constitutes no problem and does not 
change the outcome.) That is, for all $\widetilde{R_{0}} \geq 1$ the positive endemic equilibrium state $Q^{*}$ of system (3.2) exists and is globally asymptotically stable in $\Omega$. The parameter

$$
\widetilde{R_{0}}=\frac{1}{\widetilde{\delta}} \frac{\partial \widetilde{f}\left(S_{0}, I_{0}\right)}{\partial I}=\frac{1}{\delta+\alpha}\left(\frac{\partial f\left(S_{0}, I_{0}\right)}{\partial I}+p \mu\right)+\frac{\alpha}{\delta+\alpha}
$$

is not equal to the basic reproduction number $R_{0}=\frac{1}{\delta}\left(\frac{\partial f\left(S_{0}, I_{0}\right)}{\partial I}+p \mu\right)$ (van den Driessche and Watmough, 2002). However,

$$
\widetilde{R_{0}}=\frac{\delta R_{0}+\alpha}{\delta+\alpha}
$$

and it is easy to see that $\widetilde{R_{0}}$ grows with $R_{0}$, and $\widetilde{R_{0}}=1$ when $R_{0}=1$. Therefore the positive endemic equilibrium state of the system exists for all $R_{0}>1$.

As we mentioned, the feasible region $\Sigma$ is a subset of $\Omega$ and an invariant set of the system (that is a trajectory initiated in $\Sigma$ remains here), and the positive endemic equilibrium state $Q^{*}$ belongs to $\Sigma$, when it exists. Therefore, under the hypotheses of Theorem 2.1 the point $Q^{*}$ is globally asymptotically stable in $\Sigma$.

\section{Discussion AND CONCLUSION}

We proved that, under the constant population size assumption, the concavity of the nonlinear incidence rate $f(S, I)$ with respect to the number of infective host is sufficient for the global stability of the endemic equilibrium state of the $S I R$ and $S I R S$ models, and that the qualitative global behaviour of these systems is independent of how the function $f(S, I)$ depends on $S$. The Lyapunov function (2.3) suggested here make the proof almost elementary. 
This function is an extension of a Lyapunov functions constructed earlier (Korobeinikov and Maini, 2005) for the incidence rate of the form $h(S) g(I)$ to the more general case of the function $f(S, I)$. In the case of the standard bilinear incidence rate $\beta S I$ the function $V(S, I)$ takes the form

$$
V(S, I)=S-S^{*} \ln S+I-I^{*} \ln I .
$$

This function is well-known by the ecologists as the first integral and Lyapunov function for the Lotka-Volterra prey-predator system (Goh, 1980; Takeuchi, 1996), and it was also successfully applied for epidemiological models with the bilinear incidence rate (Korobeinikov and Wake, 2002; Korobeinikov, 2004,a). For the incidence rate of the form $\beta I^{p} S^{q}$ the function $V(S, I)$ takes the form

$$
V(S, I)=S+\frac{S^{*}}{q-1}\left(\frac{S^{*}}{S}\right)^{q-1}+I+\frac{I^{*}}{p-1}\left(\frac{I^{*}}{I}\right)^{p-1} .
$$

This function was constructed for SIR and SEIR models with this incidence rate (Korobeinikov and Maini, 2004) for $p<1$ (the concavity condition), and the global stability was thereby proven.

We have to remark that the concavity of the function $f(S, I)$ with respect to the variable $I$ is a sufficient but not a necessary condition for the global stability. In fact, a weaker condition (2.5) suffices to ensure the global stability (see Fig. 4.1). The monotonicity of $f(S, I)$ is not necessary either: $f(S, I)$ must ensure uniqueness of the stationary point of the function $V(S, I)$, that is uniqueness of the solution of $\frac{\partial V}{\partial S}=$ $\frac{\partial V}{\partial I}=0$, and $f(S, I)$ must grow at this point to ensure $\frac{\partial^{2} V}{\partial S^{2}}, \frac{\partial^{2} V}{\partial I^{2}}>0$ (and hence the point $Q^{*}$ is a minimum of the function $V(S, I)$ ). 


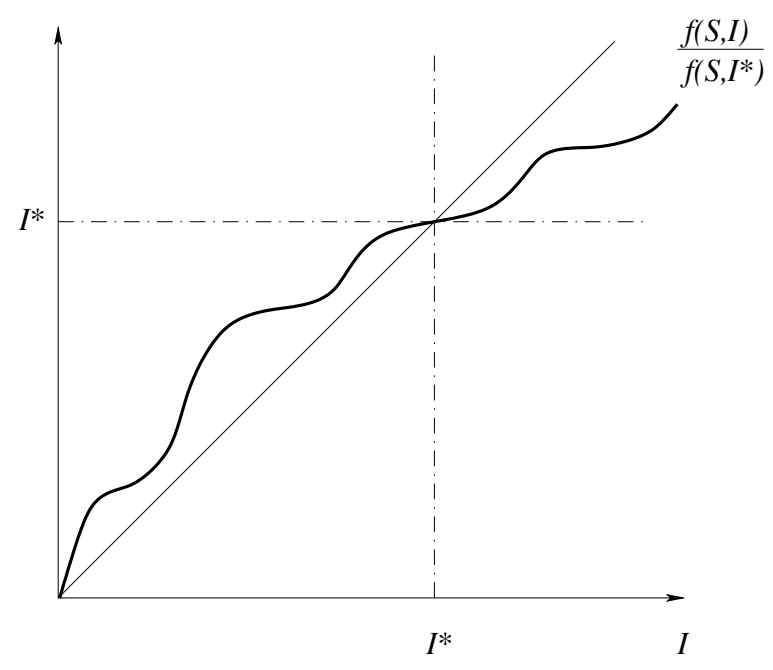

FigurE 4.1. Non-concave function $f(S, I)$ satisfying the condition (2.5).

It also follows that to have unstable nontrivial (positive) equilibria, the incidence rate $f(S, I)$ must necessarily be convex with respect to the variable $I$, at least in some points. The case of the incidence rate being convex with respect to $I$ is hardly common. Nevertheless, it may arise in some particular cases as a consequence of community effects or for a dose-dependent transmission. A system with concave incidence rate can have multiple equilibrium states and very complex behaviour (Liu et al., 1986, 1987; Regoes et al., 2002).

In this paper we postulated that the birth rate exactly equals the death rate for each compartment. Such a constraint, even if it is fairly common in the literature, may appear too restrictive. Indeed, births and deaths in a real population are not connected in any way and occur completely independently, whereas the constant population size assumption is justified by the fact that for the majority of infectious diseases epidemic processes occur on a considerably shorter timescale than the demographic process (perhaps HIV is the only exception among human diseases). 
Furthermore, for the majority of endemically persistent diseases, such as measles, chickenpox and pertussis, the class of susceptibles is composed from mainly younger people, for whom the rate of natural mortality does not necessarily coincide with that of the population as whole. For instance, in the developed countries child mortality is very low, and it can be assumed that "everyone survives to exactly age L, and then promptly dies" (Anderson and May, 1991). Such an assumption, which is known as "Type I mortality", leads to the so-called "measles model"

$$
\begin{aligned}
\dot{S} & =b-f(S, I), \\
\dot{I} & =f(S, I)-\sigma I, \\
\dot{R} & =\sigma I-b,
\end{aligned}
$$

where $b$ is the birth/dearth rate and $\sigma$ is the recovery rate.

Vaccination also leads to inequality of the birth and death rates: vaccinated hosts leave the $S$ class and move directly in the $R$ class. It may be assumed that the vaccination rate is proportional to the number of births, or to the number of the susceptible hosts $S$. However, either assumption leads to the susceptibles death rate unequal to the birth rate.

This leads to the question of whether the results obtained in this paper are robust to inequality of birth and death rates and can be applied to the more general system

$$
\dot{S}=b-f(S, I)-\mu S, \quad \dot{I}=f(S, I)-\delta I
$$


(the equation for $R$ is omitted here). The answer is affirmative: the Lyapunov function (2.3) can be straightforwardly applied to this model, with the same result.

\section{REFERENCES}

Anderson, R.M. and May, R.M. (1991) Infectious Diseases in Humans: Dynamics and Control. Oxford University Press, Oxford.

Barbashin,E.A. (1970) Introduction to the theory of stability. WoltersNoordhoff, Groningen.

Briggs, C.J. and Godfray, H.C.J. (1995) The dynamics of Insectpathogen interactions in stage-structured populations. Amer. Naturalist, 145 (6) 855-887.

Brown, G.C. and Hasibuan, R. (1995) Conidial discharge and transmission efficiency of Neozygites floridana, an Entomopathogenic fungus infecting two-spotted spider mites under laboratory conditions. Journal of invertebrate pathology, 65, 10-16.

Busenberg, S.N. and Cooke, K. (1993) Vertically transmitted diseases, Springer, Berlin.

Capasso, V. and Serio, G. (1978) A generalisation of the KermackMcKendrick deterministic epidemic model. Math. Biosci. 42, 43-61.

Derrick, W.R. and van den Driessche, P. (1993) A disease transmission model in a nonconstant population. J. Math. Biol., 31, 495-512.

Derrick, W.R. and van den Driessche, P. (2003) Homoclinic orbits in a disease transmission model with nonlinear incidence and nonconstant population. Discret Contin. Dyn. Syst. Ser. B, 3 (2) 299-309.

Feng, Z. and Thieme, H.R. (2000) Endemic models with arbitrarily distributed periods of infection I: Fundamental properties of the model. SIAM J. Appl. Math., 61 (3), 803-833. 
Feng, Z. and Thieme, H.R. (2000a) Endemic models with arbitrarily distributed periods of infection II: Fast disease dynamics and permanent recovery. SIAM J. Appl. Math., 61 (3), 983-1012.

Goh, B.-S. (1980) Management and Analysis of Biological Populations, Elsevier Science, Amsterdam.

Hethcote, H.W. (2000) The Mathematics of infectious diseases. SIAM Rev., 42 (4) 599-653.

Hethcote, H.W., Lewis, M.A. and van den Driessche, P. (1989) An epidemiological model with delay and a nonlinear incidence rate. J. Math. Biol., 27, 49-64.

Hethcote, H.W. and van den Driessche, P. (1991) Some epidemiological models with nonlinear incidence. J. Math. Biol., 29, 271-287.

Korobeinikov, A. (2004) Lyapunov functions and global properties for SEIR and SEIS epidemic models. MMB IMA, 21, 75-83.

Korobeinikov, A. (2004a) Global properties of basic virus dynamics models, Bulletin for Mathematical Biology, 66 (4), 879-883.

Korobeinikov, A. and Maini, P.K. (2004) A Lyapunov function and global properties for SIR and SEIR epidemiological models with nonlinear incidence. Math. Biosci. Engineering, 1 (1), 57-60.

Korobeinikov, A. and Maini, P.K. (2005) Nonlinear incidence and stability of infectious disease models, MMB IMA, 22, 113-128.

Korobeinikov, A. and Wake, G.C. (2002) Lyapunov functions and global stability for SIR, SIRS and SIS epidemiological models. Appl. Math. Lett., 15 (8), 955-961.

La Salle, J. and Lefschetz, S. (1961) Stability by Liapunov's direct method. Academic Press, New York. 
Li, M.Y., Muldowney, J.S. and van den Driessche, P. (1999) Global stability of SEIRS models in epidemiology, Canadian Appl. Math. Quort. 7.

Liu, W.M., Levin, S.A. and Isawa, Y. (1986) Influence of nonlinear incidence rates upon the behaviour of SIRS epidemiological models. J. Math. Biol., 23, 187-204.

Liu, W.M., Hethcote, H.W. and Levin, S.A. (1987) Dynamical behavior of epidemiological models with nonlinear incidence rates. J. Math. Biol., 25, 359-380.

Regoes, R.R., Elbert, D. and Bonhoeffer, S. (2002) Dose-dependent infection rates of parasites produce the Allee effect in epidemiology, Proc. R. Soc. Lond. B, 269, 271-279.

Takeuchi, Y. (1996) Global Dynamical Properties of Lotka-Volterra Systems, World Scientific, Singapore.

van den Driessche, P. and Watmough, J. (2002) Reproduction numbers and sub-threshold endemic equilibria for compartmental models of disease transmission. Math. Biosci. 180, 29-48. 